\title{
VIDA HUMANA, INTERRUPCIÓN Y CESE
}

\section{Osvaldo Nelo Tieghi}

Presidente de la Asociación de Criminología, Argentina

\section{1) Anticipo de conclusiones (De jure condendo):}

$\left.1^{\circ}\right)$ Consideramos que doctrinario y legislativamente debe distinguirse entre: 1 . los «métodos y prácticas de reanimación " que tienen por objeto el "auxilio" de la «naturaleza espontánea de las funciones inherentes a la vida del hombre» (v. gr., respiración y circulación), cuando no haya tenido lugar el "cese irreversible y total» de aquéllas, con inequívoca significación de "muerte real o médico-legal» y 2 . las técnicas subsiguientes a las llamadas "muerte cerebral» y "encefálico", dirigidas a mantener únicamente la actividad de algunos órganos, ( $p$. ej. el corazón) confines utilitarios.

En los fines o metas de tales técnicas - a más de la correspondencia entre el diagnóstico - médico y su materialidad verdadera, esto es, de la comprobación de la conformidad entre el primero y la última -, habrá de descubrise y desvelarse al criminal homicida o al terapeuta, al menos, sí la moral fundamenta la ley: léanse reglamentaciones bioético - jurídicas de trasplantes (cfr., asimismo, Domingo $\mathrm{M}$. Basso, Nacer y morir con dignidad. Bioética, 426, 427, 429, 430, 433, 436, 439, etc.; Avelino V. Do Pico y otros, en La certificación de la muerte ante la ley de trasplantes, La Ley, 1982, D-741 y ss.; Carlos H. Vidal Taquini, Muerte real y muerte clínica, La Ley, 1980-C-1066 y ss.; Martín Anzoategui, Problemas penales de los trasplantes cardíacos, La Ley, 135-1614 y ss.).

$\left.2^{\circ}\right)$ Los métodos mecánicos para el «mantenimiento de órganos destinados a trasplantes" no podrán en ningún caso utilizarse sobre la persona que aún no ha sufrido «muerte real», debidamente certificada, sin que ningún proceso anterior (v. gr., muerte cortical, muerte cerebral o encefálico o coma irreversible) pueda legitimarlos.

En caso contrario, tales maniobras deberán ser objeto de tipificación y sanción penal (cfr., p. ej., E. F. P.Bonnet, Medicina..., 1, 279 y ss.; Do Pico, Riú, Cortecci y Bellani, La certificación..., 749, 750 y 752; María T. Bergoglio de Brouwer y María V. Bertoidi, La eutanasia, distanasia y ortotanasia..., en El Derecho 117-780 y ss.; Domingo M. Basso, ob. cit., 439).

3\%) El retiro de los instrumentos de «mantenimiento artificial de vida vegetativa", cuando aquéllos han sido instalados contraviniendo lo prescripto en los dos números anteriores, constituye, a nuestro juicio, delito de homicidio contra la vida de la persona sujeta a tales prácticas y debe evitarse la legitimación de tales hechos a través de las leyes de trasplantes (Cfr., también, Domingo M. Basso, cit., 439.; Do Pico y otros, ob. cit., 752). 
$4^{2}$ ) Aun cuando el hecho ya se encuentra regulado en los diversos Códigos penales o en las leyes especiales, debe tenerse presente y reiterarse, aquí, que las prácticas de reanimación artificial deben considerarse obligatorios tanto en los casos de primeros auxilios, como cuando lo requiera la salud del paciente para la eventual activación de sus funciones cardiocirculatorias o respiratorias, en cualquier etapa de un tratamiento o intervención quirúrgica (cfr., asimismo, Domingo Basso, ob. cit., 440).

$\left.5^{2}\right)$ Pero el uso de técnicas reanimativas o soportes artificiales, puede tener lugar mas allá de todo requerimiento razonable, como una suerte de encarnizamiento o ensañamiento terapéutico, cuando la tecnología y los conocimientos de su tiempo permiten reputarlos - en acuerdo médico general - como absolutamente inútiles e inoperantes; en tal caso, deben ser rechazados por su carácter moralmente ilícito y, eventualmente, deberán ser sancionados por la ley penal si se acreditaren propósitos de lucro o intenciones relativas al logro de trasplante de órganos.

$\left.6^{\circ}\right)$ No debe considerarse moralmente ilícita, consecuentemente con lo dicho en el número anterior, ni sujeta a sanción penal alguna, la conducta de quienes hubiesen dispuesto y aceptado, respectivamente, la suspensión de prácticas y técnicas de reanimación desproporcionadas o extraordinarias, esto es, cuando ya no existiesen esperanzas de resultado alguno de acuerdo a los medios y conocimientos de la época en que ello ocurre y sí, sólo, otros (ob cit., 752) quienes acertadamente observan: "La muerte cerebral, encefálico, del tronco encefálico o clínica es una muerte aparente, no real, irreversible a veces, pero parcial. Esta muerte utilitaria, permite extraer, sobre todo, el corazón, cuando aún no ha dejado de latir. Es una muerte por conveniencia, por acuerdo de un grupo de médicos. Considerarnos que la extracción del corazón y la consiguiente muerte de seres que han dejado de tener valor social, como los que presentan muerte cerebral, debe ser considerada como homicidio por cuanto no objetivan el paro cardiorespiratorio irreversible y completo...".

Añadamos aún, a lo señalado, que el propio Bonnet, en la obra citada $(1,289)$ refiriendo al "coma sobrepasado" - en el cual se produce la abolición total de las funciones de la vida de relación, y de las funciones de la vida vegetativa - advierte cómo los autores de la nueva variedad de "coma" transforman en regla absoluta una posibilidad: la de hacer equivalentes la interrupción del control cardiocirculatorio y la de la muerte real, asimismo, otorgan al médico el derecho (es prácticamente un derecho omnipotente y divino) de decidir cuándo la sobrevida se interrumpirá y el paciente deberá morir, morir de verdad".

Luego veremos si la temporal ley de trasplantes - poniendo aquí como ejemplo a la 24.193 que se halla vigente en Argentina - ha podido compatibilizar las leyes divinas, las de la moral, y las de la naturaleza, con la "relatividad instrumental», la "perfectibilidad" y la "falibilidad científica" de los conceptos médicos sobre "predictibilidad" del "proceso mortal".

Pues bien, pese a esta "relatividad cognitiva", que subordina todo conocimiento científico - experimental, bajo control de variables, al estadio instrumental de su época (que ni siquiera es el caso de esta simple prognosis empírica médica) algunos juristas argentinos han Ilamado "cadáveres humanos» sujetos a trasplantes (art. 1' de la Ley 24193) a los referidos supuestos de "Muerte cerebral». Podría decirse que se han creado dos clases de muerte: una muerte cerebral, para los donantes; otra, la común, para los no trasplantados. Como señala Ricardo D. Ravinovich (Régimen..., 67) esto 
parece natural porque el muerto cardíaco es un pobre donante ya que sus órganos dejan enseguida de ser útiles. En expectancias de lucro o experimentación o cualquiera otra ajena a la razonable mejoría de paciente (cfr., asimismo, Declaración sobre...).

\section{Concepto de muerte:}

\section{a) Generalidades y muerte en sentido jurídico o médico legal.}

Se considera tal a aquella que se constata en los términos de cada legislación para el asiento respectivo ante el Registro Civil. En términos de Bonnet esta es la que tiene lugar - biológicamente - por el cese de la función cardíaca, con el consecuente paro de la circulación sanguínea, y el de la oxigenación viscerohístico - celular y, por tanto, la supresión de la respiración (Medicina legal, 1, 282).

Ya en la medicina egipcia se atendía a la paralización de la respiración, la cual era sucedida por la del corazón.

Sobre fines del siglo XVII Marie Francois Xavier Bichat pone el acento en la detección definitiva del trípode respiratorio, cardiocirculatorio y nervioso.

Para entender la materia bajo tratamiento debe comprenderse que existe un ciclo o proceso mortal cuya interrupción se pretende legitimar con nominaciones diagnósticas que no exceden a un mero pronóstico anticipado a la muerte real (cfr., Vidal Taquini, ob cit., 1066 y ss.): Si una persona se encuentra con funcionamiento respiratorio y cardíaco, debido a que es asistido o mantenido por medios o procedimientos electromecánicos de reanimación, o extracorpóreos, este individuo, desde el punto de vista médico - legal se encuentra vivo $v$ esta supervivencia y auxilio artificial debe cesar, desde el punto de vista ético - médico, sólo cuando ya no hay posibilidades razonables de recuperación natural o espontánea..." (Avelino V. Do Pico y otros, ob.cit., ps. 741 y ss.).

Antes de ingresar en los conceptos correspondientes a la denominada muerte cerebral o encefálico - cuyo término ha sido y es seriamente cuestionado en cuanto se le aplica para referir a alguna instancia de muerte (cfr., v. gr., José W. Tobías, Fin de la existencia..., 20 y ss.; Alberto Bueres, Responsabilidad..., 327 y 328; Basso, ob cit., 430 y 431) vale la pena recordar la opinión, destacable, entre otros, de Do Pico y términos de Maas y Danielián (El derecho a morir con dignidad, 856) se ha institucionalizado la agonía como paso intermedio y obligatorio entre la vida y la muerte.

\section{b) Muerte fisiológica y muerte biológica:}

Se denomina como muerte fisiológica a aquélla que tiene lugar por simple envejecimiento. Acaece, como bien señala Basso por las mutaciones normales ocurridas fatalmente a nivel celular (ob. cit. 428). No ocurre tal muerte, en consecuencia, por causa de una patología o enfermedad concreta. La muerte patológica, en cambio, halla su etiogénesis en causas mórbidas. 
c) Muerte relativa, intermedia y absoluta:

Mantovani, en su Enciclopedia dil diritto (XXVII, 83), establece estas tres fases del proceso mortal: "Muerte relativa», cuando tiene lugar el cese de las funciones nerviosa, cardiocirculatoria y respiratoria; "Muerte intermedia», que ocurre cuando tales funciones quedan irreversiblemente detenidas y muerte absoluta o biológica, en la cual hay cesación de cualquier clase de vida celular teniendo lugar la ausencia definitiva de toda actividad biológica.

\section{d) Fijación clínica de la muerte:}

La cuestión que debe resolver la medicina - en la observación directa de los pacientes - es en qué etapa del proceso anterior a la "absoluta" debe considerar operada la muerte. Se ha descartado la necesidad de esperar el estadio de la muerte real o absoluta. Así la muerte clínica precede a aquélla.

En principio la muerte clínica coincidió con la supresión de las funciones respiratorias; luego, con el cese del latido cardíaco; finalmente, con la pérdida de las tres funciones vitales: la respiratoria, la cardiocirculatoria y la nerviosa.

\section{e) Muerte aparente y biológica:}

Según Camilo Simonín (732) la muerte aparente es aquélla en la cual las funciones vitales están en suspenso. Pese a la detención momentánea de éstas, con movimientos cardíacos clínicamente imperceptibles, a la inmovilidad, inconsciencia y falta de respuesta sensoriales, las pruebas demuestran que el individuo, a un mínimo, mantiene tales funciones.

La muerte biológica o absoluta, como vimos, es la de células que componen los tejidos. En términos de Do Pico (ob. cit., 743) el individuo va muriendo poco a poco, a medida que lo van haciendo las células y tejidos. Las últimas células en morir son las epiteliales que tienen una vitalidad de alrededor de 24 horas. El óvulo vive 24 horas, los espermatozoides, tres días (dentro de las trompas); las córneas 48 horas, colocadas en solución fisiológica; el músculo cardíaco, ya paralizado tiene una vitalidad de 20 a 30 minutos. Si se lo somete a una hipotermia simple - temperatura un poco superior al punto de solidificación - 4 grados centígrados, tiene una vitalidad de tres horas, etc. (ob. cit. 743).

\section{j) Muerte cerebral y conclusiones :}

Como bien señala Basso y otros autores (v. gr., Tobías, 15 y ss.; Bueres, 327; Bonnet, 1, 288 y ss.; Do Pico, Riu y otros, 744; Bustamante Alsina, 1338 y ss.; Maas y Danielián, 856) la muerte cerebral es una muerte utilitaria que ha nacido a la luz de las modernas técnicas de reanimación (Basso, cit., 428 y 429), más aparente que real, irreversible a veces y que permite extraer el corazón cuando aún no ha dejado de latir: una muerte por conveniencia gracias al acuerdo de un grupo de médicos (Do Pico, Riu y otros, cit., 752). 
Gran cantidad de países aceptaron el fallecimiento por «muerte cerebral»o "paro irreversible de la funciones cerebrales», en la cual se detecta, por EEG el silencio cerebral a través del trazado isoeléctrico, cuyos tiempos han sido fijados en forma absolutamente variada, esto es entre los 30 minutos y las 48 horas, hecho objeto de observación y crítica por parte de Bonnet.

Ya a mediados de 1985, 32 estados de Norteamérica receptaron legislativamente como diagnóstico de causa de muerte a la cesación irreversible de la vida cerebral.

Casi tan terminante como Riú y Do Pico se pronuncia el prestigioso médico legista Bonnet (288 y ss.), para quien esta forma de diagnóstico no tuvo por fin establecer la muerte real o verdadera ya que su origen y finalidad fueron otras: "Poder continuar, una vez superado el escollo administrativo del certificado de defunción, con la circulación artificial (por lo demás no interrumpida en ningún momento), hasta el instante adecuado para que "en caliente " se retire el corazón a esa persona " (agregamos nosotros: a la que la ley de trasplantes indebidamente llama cadáver) para insertársela a otra.

Se ha buscado refugio de certeza predictiva; pero ello no es aceptable, ante la posible superación instrumental, aun en la verificación rigurosa y científica, cualquiera sean las variables controladas en su tiempo (salvo para esa época, en relación con esas variables y ese instrumental; cfr., Tieghi, Tratado..., 142 y ss.).

El caso de Karen Ann Quinlan, en el cual la Suprema Corte de New Yersey permitió a la familia de la paciente retirarle el respirador artificial que parecía auxiliarle en estado vegetativo desde hacía siete años, dejó en claro la relatividad de todo pronóstico de muerte; en efecto Quinlan sobrevivió nueve años al retiro del respirador (cfr., Montoya, El derecho..., 1065 y ss.).

Ya hemos dicho anteriormente, en los puntos $1^{\circ}$ y $2^{\circ}$ del Anticipo de conclusiones que las: prácticas de reanimación producidas con anterioridad a la muerte real (cuando ya ha ocurrido el cese de la actividad cardíaca, circulatoria y respiratoria) con meros fines de preservación para trasplantes deben prohibirse; asimismo que dicha reanimación artificial no puede permitirse más allá de las posibilidades razonables de recuperación espontánea de esos centros cardio - respiratorios.

Deben merituarse cuidadosamente las observaciones que efectúan Adams, Raymond y Maurice, Victor en sus Principios de neurología (802), quienes enseñan que en el síndrome de muerte cerebral, las perspectivas de recuperación, que, sin duda, existen, son escasas y que se debe valorar, prudentemente, la posibilidad de suprimir las medidas de sostén (respiración asistida, agentes vaso depresores, etc.).

En efecto, añaden los autores, estos pacientes a menudo se convierten en víctimas donantes de órganos: "...debe tenerse mucho cuidado a la hora de asegurar que el paciente sufre un "daño cerebral irreversible», puesto que la "antestesia", intoxicación por drogas, y la "hipotermia" pueden dar lugar también al coma profundo y a EEG isoeléctrico, pero permiten la recuperación..."

Coincidimos con Do Pico (752) cuando señala: "...consideramos que la extracción del corazón y la consiguiente muerte de seres a los que se considera que han dejado de tener valor social, como los que presentan muerte cerebral, debe ser 
considerada homicidio, pensando de esta manera, no deberíamos tener inconveniente que cuando se hace diagnóstico de agonía muy prolongada, incierta o dolorosa se pueda requerir, que la eutanasia sea incluída entonces, como justificante sin cometer homicidio y así iremos entrando bajo el concepto de adelanto de la medicina en una serie de concesiones de cuya peligrosidad no podemos, siquiera, llegar a imaginar..."

Repugna a la sensibilidad humana, más allá de los cuestionamientos jurídico científicos, jurídico - médicos y jurídico - religiosos que, con el corazón latiendo, el hombre quite la vida de aquel semejante al cual él mismo se la prolongó mercantilmente, sobrevalorando el límite de sus conocimientos y lo que es peor, aún, que la ley legitime estas manipulaciones y declare cadáver a quien, aún vegetativamente, vive (cfr., arts. 1 y 23 de la Ley 24.193).

\section{3) Declaraciones diversas sobre muerte cerebral:}

En general, y atendiendo al hecho de que las modernas técnicas de reanimación se interponen entre la llamada "muerte cerebral» y la "muerte verdadera», "real» 0 "absoluta", impidiendo la comprobación de esta última -esto es el efectivo "paro cardíaco" y "cese circulatorio, respiratorio" y de la "actividad cerebral», independientemente de la referida "asistencia artificial ininterrumpida"se han propuesto múltiples exámenes que a nuestro juicio no obstan a la referida interrupción intencional del proceso presuntamente mortal.

La Declaración de Sydney, o mas bien de la Asociación Médica Mundial con sede en Sydney, revisada en Venecia en 1983, atendió a la muerte cerebral y encefálico. Ciccone (Questioni..., 152), citado por Basso, añade: "El cerebro puede decirse muerto cuando ya no funciona en su totalidad, aún en los centros de la vida_vegetativa. La muerte de éstos resulta del hecho de que ya no existe posibilidad de restablecer una respiración y circulaçión espontáneas y autónomas... "

Entre los puntos más significativos de la denominada Declaración de Sydney destacamos los siguientes:

$1^{\circ}$ ) "La cuestión del momento de la muerte de las diferentes células y órganos es aquí menos importante que la certeza de que este proceso se ha tornado irreversible, sean cuales fueren los métodos de resucitación que puedan ser empleados".

$2^{\circ}$ ) "No existe en el estado actual de los conocimientos médicos, criterio único que sea plenamente satisfactorio y ningún procedimiento técnico actual podrá reemplazar al criterio clínico del médico",

3ㅇ) "La determinación del momento de la muerte permitirá, desde el punto de vista ético, cesar los esfuerzos de reanimación, y en los países en los que la ley lo permite, efectuar la extracción de los órganos".

Aunque no coincidimos con los autores que consideran que la muerte es un «instante» - ya que considerarnos que la vida y la muerte constituyen un todo dinamogenético en "curso" o "proceso"- creemos, como muchos de aquellos, que la "anticipación clínica» a la "muerte absoluta» o «real» constituye homicidio (cfr., Vidal Taquini, cit., 1066 y ss.; Bonnet, cit., 279 y 289; Do Pico, Riú, Cortecci y Bellani, cit., 
741 y ss., entre otros), y que el "paro cardíaco» $y$ "respiratorio» es el punto final a tal proceso y el instante mismo de la "muerte real».

Los innumerables casos de incertidumbres fatales sobre personas enterradas vivas y que después de inhumanadas dejaron las señales inequivocas de Infalibilidad clínica, aún en épocas no contaminadas por los trasplantes, y también hasta los últimos años (cfr., asimismo, Diccionario razonado .. ), se suman a los sucesos múltiples de «reanimación espontánea» relatados por Bernardo E. Malamud, 1066 y ss.).

Euclides Zerbini y tantos otros (cfr., Bueres, cit., 327 y 328 y Vidal Taquini, cit.,

Creemos que deben descartarse y transcribirse aquí unos párrafos inapreciables de Bonnet (cit., I, 279): "Quien es mantenido en "circulación extracorpórea", pese a estar in artículo mortis, no es un cadáver. Cuántos lo han estado, cuántos han sido desahuciados - hemos visto sujetos estar veinte días en estado de coma, y recuperarse y seguir viviendo - pero no por ello se les firmó un certificado de defunción anticipado. En cambio, el candidato al trasplante - Con el pretexto de la necesidad de una víscera en estado óptimo - fue declarado muerto sin estarlo realmente, y lo que es más grave, sin saber nadie, absolutamente nadie, si inmediatamente después de habérselo declarado tal, en virtud de sus reservas orgánicas, podía recuperarse".

Como bien señala Basso, junto con la técnica de los trasplantes, ha aparecido otro nivel de estado de coma denominado depassé en el cual se puede afirmar que un organismo humano, de hecho muerto por la cesación total y definitiva del sistema nervioso central es mantenido artificialmente y preservado de las consecuencias degenerativas de sus órganos, asegurándole la irrigación con sangre oxigenada, mantenida forzadamente en circulación. En este caso la muerte cerebral no ocurre naturalmente. La crean los médicos a través de la tecnología terapéutica (Basso, ob. cit., 430).

Conforme reproduce Basso (cit., 432) la Pontificia Academia de Ciencias ha opinado que la muerte sobreviene cuando: 1' La funciones espontáneas cardíacas y respiratorias cesaron definitivamente; 2' Se verificó que el cerebro no presenta actividad eléctrica alguna mediante un exámen doble con una distancia de seis horas.

El II Congreso de la Sociedad Italiana de Trasplantes, celebrado en San Remo, en 1968, estableció la diagnosis precoz de muerte en los casos siguientes: $1^{\circ}$ Coma Profundo con atonía muscular; $2^{\circ}$ Ausencia de respiración espontánea, después de suspender la artificial; $3^{\circ}$ Cese de actividad eléctrica: por 24 horas, en caso de coma por lesión encefálico primitiva; por 48 horas, para los supuestos de lesión encefálico secundaria.

En la prueba relativa a la falta de respiración espontánea, que obviamente no podrá pretenderse en quienes se hallan conectados al respirador artificial, salvo su suspensión, como se propuso correctamente en San Remo, debe tenerse presente lo que señalan Do Pico, Riú, Cortecci y Bellani: "En dicha prueba la falta de respiración podría también deberse, como se observa en ciertos traumatizados de cráneo, al edema cerebro bulbar, que con tratamiento adecuado podría retrogradar en horas o días". Los autores citan, entre otros supuestos, el caso de Karen Quinlan. 
El artículo 23 de la Ley 24.193 de Trasplante de órganos establece que el fallecimiento de una persona se considerará tal cuando se verifiquen de modo acumulativo los siguientes signos, que deberán persistir ininterrumpidamente seis horas después de su constatación conjunta: a) Ausencia irreversible de respuesta cerebral, con pérdida absoluta de conciencia; b) Ausencia de respiración espontánea; c) Ausencia de reflejos cefálicos y constatación de pupilas fijas no reactivas; d) Inactividad encefálico corroborada por medios técnicos y/o instrumentales adecuados a las diversas situaciones clínicas, cuya nómina será periódicamente actualizada por el Ministerio de Salud y Acción Social con el asesoramiento del Instituto Nacional Central único Coordinador de Ablación e Implante (INCUCAI). La verificación de los signos referidos en el inc. d no será necesaria en caso de paro cardiorespiratorio total e irreversible.

\section{BIBLIOGRAFIA CITADA}

ADAMS, Raymond D. y VICTOR, Maurice, Principios de Neurología, Reverté, Barcelona, 1981.

ANZOATEGUI, Martín, Problemas penales de los trasplantes cardíacos, Rev. La Ley, t. 135, ps. 1614 y ss.

BASSO, Domingo M., Nacer y morir con dignidad. Bioética, Corporación de abogados católicos, Bs. As., 1993.

BERTOGLIO DE BROUWER de KONING, María T. y BERTOLDI, María V., La eutanasia, distanasia y ortotanasia. Nuevos enfoques de una antigua cuestión, en Rev. El Derecho, t. 117 , ps. 780 y ss.

BONNET, E.F.P., Medicina Legal, López editores, Bs. As., 1980. 1979 .

BUERES, Alberto J., Responsabilidad civil de los médicos, Abaco, Bs. As.,

BUSTAMANTE ALSINA, Jorge, Determinación del momento de la muerte y la presunción legal del consentimiento del dador en el trasplante cadavérico de órganos, en Rev. La Ley, t. 1994-E, ps, 1338 y ss.

CICCONE, L., Non uccidere. Questioni de morale della vita física, Ares., Roma, 1984; Salute e malatia. Questioni de morale della vita física, 2ª ed., Ares, Milán, 1986.

Diccionario Razonado de Legislación y Jurisprudencia, París, 1869.

DO PICO, Avelino, RIU, Jorge A., CORTECCI, Lydia y BELLANI, María Elena, La certificación de la muerte ante la Ley de trasplantes (art 21 de la reglamentación de la ley 21.541). Objeciones médico legales, en Rev. La Ley, t. 1982-D, ps. 741 y ss.

MAAS, Noel y DANIIELIAN, Miguel El derecho a morir con dignidad, Rev. El Derecho, t. 89, ps.855 y ss.; Prohibido experimentar con el hombre, Rev. El Derecho, 
t. 120 , ps. 943 y ss.; Los inciertos extremos de la existencia humana, en Rev. El Derecho, t. 105, ps. 787 y ss.

MANTOVANI, Fernando, Enciclopedia dil diritto, Milano, Giuffré.

MONTOYA, Mario Daniel El derecho constitucional a morir, en Rev. La Ley, t. 1991 -A, ps. 1065 y ss.

RABINOVICH, Ricardo David, Régimen de trasplantes de órganos y materiales anatómicos. Ley 24.193, Astrea, Bs. As., 1994.

SIMONIN, Camilo, Medicina legal, Jims editor, Barcelona, 1980. 1996.

TIEGHI, Osvaldo Nelo, Tratado de criminología, Universidad, Bs. As., $2^{\circ}$ ed., 1988.

TOBIAS, José W., Fin de la existencia de las personas físicas, Astrea, Bs. As.,

VIDAL TAQUIM, Carlos H., Muerte real y muerte clínica, en Rev. La Ley, t. 1980-C, ps. 1066 y ss. 